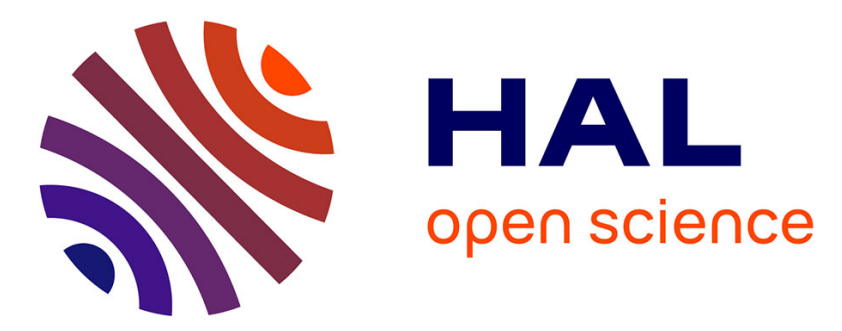

\title{
Robust Virtual Keyboard for Brain-Computer Interface (ROBIK): An Halfway Update on the Project
}

\author{
Louis Mayaud, Marco Congedo, Sabine Filipe, Guillaume Charvet, Remi
}

Schoettel, Djillali Annane

\section{- To cite this version:}

Louis Mayaud, Marco Congedo, Sabine Filipe, Guillaume Charvet, Remi Schoettel, et al.. Robust Virtual Keyboard for Brain-Computer Interface (ROBIK): An Halfway Update on the Project. Second IASTED International Conference on Robotics (Robo 2011), Nov 2011, Pittsburg, United States. pp.752-007. hal-00728525

\section{HAL Id: hal-00728525 \\ https://hal.science/hal-00728525}

Submitted on 6 Sep 2012

HAL is a multi-disciplinary open access archive for the deposit and dissemination of scientific research documents, whether they are published or not. The documents may come from teaching and research institutions in France or abroad, or from public or private research centers.
L'archive ouverte pluridisciplinaire HAL, est destinée au dépôt et à la diffusion de documents scientifiques de niveau recherche, publiés ou non, émanant des établissements d'enseignement et de recherche français ou étrangers, des laboratoires publics ou privés. 


\section{ROBUST VIRTUAL KEYBOARD FOR BRAIN-COMPUTER INTERFACE (ROBIK): AN HALFWAY UPDATE ON THE PROJECT}

\author{
Louis Mayaud \\ IBME, University of Oxford \\ Old road Campus Building, OX3 7DQ Oxford \\ United Kingdom \\ louis.mayaud@eng.ox.ac.uk \\ Tel: +447984714785
}

Guillaume Charvet

CEA-Leti

17 , rue des Martyrs

38054 Grenoble, France

guillaume.charvet@cea.fr

Tel: +33438780144

\author{
Marco Congedo \\ Gipsa-Lab CNRS \\ 961, rue de la Houille Blanche \\ 38402 Grenoble, France \\ marco.congedo@gmail.com \\ Tel: +33476826252
}

\author{
Remy Schoettel \\ DIXIMicrotechniques \\ 4, rue chemin de Palente \\ 25025 Besançon, France \\ r.schoettel@diximicrotechniques.com \\ Tel: +33381889890
}

Sabine Filipe

CEA-Leti

17, rue des Martyrs

38054 Grenoble, France

sabine.filipe@cea.fr

Tel: +33438780144

Djillali Annane

CICIT-APHP

104, boulevard Raymond Poincar

92380 Garches, France

djillali.annane@gmail.com

Tel: +33147104474

\section{ABSTRACT}

Introduction The principle of a Brain-Computer Interface or $\mathrm{BCI}$ is to control a device through the extraction and interpretation of signal features from electroencephalograms (EEG) collected either from the surface of the scalp or through invasive measurements. This late idea of communication technique (Vidal 1973), offers the advantage of bypassing the need for muscle activity in the control chain and is therefore presented as a promising alternative to restore communication and control in severely disabled patients (Wolpow, et al. 2002). However, the lack of robustness and ergonomics of both available software and EEG measurement techniques have delayed the transfer of this technology to patients bedsides.

Materials and methods The French Research Agency has funded a 3-year project gathering national leaders in microelectronics (CEA-Leti), EEG signal processing (Gipsa-Lab) and clinical management of severely disabled people (Raymond Poincar hospital). The aim of the project is the development and the clinical validation of a BrainComputer Interface prototype for communication. As an initial step, a survey was carried out to assess patients' and users (family and caretakers) needs, which were translated into specifications, on the basis of which software and hardware were developed.

Results The survey ( $\mathrm{n}=45)$ highlighted the need for easy-to-setup systems (installation time ; $15 \mathrm{~min}$ ), which stresses the importance of mechanical comfort and customization of application. The development of signal processing techniques has led to improvements of the P3Speller paradigm. A first prototype of a 32-channel EEG recording system is under development. To ease the EEG measurements and reduce installation time, the system has a reduced size. It includes the analog amplification and digital conversion of 32 channels sampled at $1 \mathrm{kHz}$, as well as the wireless data transmission to a computer. First in vivo validations were performed on small animals. This system will be optimized and connected to a headset specifically designed to provide a comfortable and handy interface with dry electrodes.

Discussion The present project will still run for one and a half years, ending with its clinical validation in a population of severely disabled patients, which will compare performances of the system with existing assistive technologies. At this stage, the proposed system yields very promising results, and outperforms the current state-of-theart. If such a system is shown to perform better than current users assistive technology, it could reach the commercial availability for severely disabled patients within the next 5 years.

\section{KEY WORDS}

Brain-Computer Interface (BCI), electroencephalography (EEG), Evoked Potentials, dry electrodes, assistive technology, P300 speller

\section{Introduction}

The principle of a Brain-Computer Interface (BCI) is to control a device through the extraction and interpretation of signal features from electroencephalograms (EEG) collected either from the surface of the scalp or through invasive measurements. This old idea of communication technique [1], offers the advantage of bypassing the need for muscle activity in the chain of control, and is therefore presented as a promising alternative to restore communication and control in severely disabled people diseases $[2,3]$.

The use of BCI in a severely disabled population is however less frequent than commonly believed. Firstly, a literature research on BCI shows that a third of published papers deal with algorithms aiming to provide higher and more robust overall performances. Unfortunately, only a few algorithms are made available on one of the two open-source platforms for BCI $[4,5]$. In fact, only two 
implementations of the well-known and broadly-published P3Speller [6] make use of these platforms. Secondly, nearly a quarter of the publications focus on neurophysiology and the use of EEG features with no major breakthrough in this direction, as BCI mainly makes use of late paradigms: Event Related Potentials (ERP) [7, 8], Steady State Visual Evoked Potentials (SSVEP) [9], motor rhythms [10] and Local Field Potentials (LFP) [11]. Finally, only a fifth and a tenth of publications deal with specific applications and evaluation in patients, respectively. Before 2005, the proportion of medical publications in the field was around $10 \%$, a figure that nearly doubled since then.

The Clinical Investigation Centre for Innovative Technology (CIC-IT) at the Raymond Poincar hospital (Paris hospital trust - APHP) is the leading French multidisciplinary research group in the assessment of technology for use by disabled patients. BCI was lately identified as a potential assistive technology, despite being regarded with much skepticism by clinicians, due to the disadvantageous usability-performance ratio against existing assistive technologies. Further examination of the reasons why BCI are not being widely available led to the following conclusions:

- Lack of robustness of the application: no real adaptive signal processing tools taking into account the interand intra-individual variations of EEG features and high sensitivity to all sources of noise ;

- Lack of ergonomics: the setup procedures of EEG recording device is particularly tedious. Moreover, the software calibration procedures (primarily designed for researchers) are complex and are not integrated with end-user application such as emails or Internet.

A consortium was built and a project proposal developed whose aim is to investigate these issues, while working on hardware and software simultaneously, in close relation with patients and medical teams specifications. Finally, the French Research Agency funded a 3year project regrouping national leaders in microelectronics (CEA-Leti), EEG signal processing (Gipsa-Lab) and clinical management of severely disabled people (Raymond Poincar hospital) together with an industrial leader in invasive brain signal monitoring (DIXImicrotechniques). The project aims to develop and validate a BCI prototype for communication. As an initial step, a survey was carried out to assess patients and users (family and caretakers) needs, which were translated into specifications, subsequently used as guidelines for both software and hardware development.

\section{Materials and methods}

\subsection{Patients need and technological locks}

A questionnaire was written by occupational therapists specialized in providing effective assistive technology to a

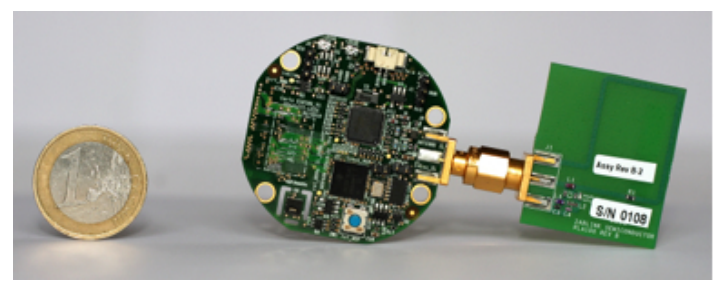

Figure 1. Picture of the ECRINS electronic board with its RF antenna used for the RoBIK project.

wide range of disabled patients. The questionnaire was composed of three sections: general use of BCI, BCI headset, and application. 20 patients and 20 caretakers were interviewed. Quadriplegic patients admitted to ICU for whom verbal communication was compromised by mechanical ventilation, were enrolled after informed consent. The aim of the clinical study is to assess the feasibility of a state-of-the-art BCI for communication described in [12]. 24 cupule-electrodes were fitted to 10/20 standard locations by an EEG technician and signals were recorded at 256 $\mathrm{Hz}$ with a Porti32 ${ }^{T M}$ from TMSi (Twente, Netherlands). The Porti32 $2^{T M}$ was connected to the open-source platform OpenViBE. Signals were filtered using the xDAWN spatial filter [13] prior to SVM voting classifier [14].

\subsection{New P300 paradigm for daily use}

Our cohort consists of 20 healthy volunteers who gave their informed consent. For each of them, 32-EEG signals were recorded using a Nexus $32^{T M}$ from MindMedia (Roarmond-Herten, Netherlands). Recordings took place in a room specifically labeled by the national health and safety regulatory committee (AFSSAPS). Subjects were given instructions for a standard P3Speller experiment [12]. Stimulation and signal processing were handled by OpenViBE. Signals were analyzed with the aim of optimizing:

- the number and locations of sensors ;

- pre-processing and classification parameters.

\subsection{Wireless EEG headset for daily use at home}

A wireless multichannel data acquisition electronic system is being designed for electroencephalograms (EEG) recording. This system is based on the EleCtro encephalogramme Recording Interface for Neuroactivity Studies (ECRINS) prototype which is shown on the Figure 1. This electronic system is consists of a custom integrated circuit (ASIC) for signal conditioning, amplification and digitization, as well as a commercial module for RF transmission. The RF transmission module is capable of handling a 32-channel EEG recording sampled at $1 \mathrm{kHz}$ with 12-bit resolution. This integration is the first step towards a lightweight EEG headset for Brain Computer Interface (BCI) studies. 
In parallel, an extensive literature review for EEG sensors was carried out. Three dry-electrodes solutions for easy-to-setup BCI headsets were identified: micro-array electrodes [15], comb electrodes [16, 17] and hybrid electrodes [18]. Commercially available solutions are currently evaluated together with a prototype developed by the projects industrial partner. Eventually the selected candidate will be interfaced with an EEG headset designed to comply with specifications. To date, several headset designs were suggested and are currently prototyped.

\section{Results}

\subsection{Patients need and technological locks}

The survey ( $\mathrm{n}=40$ ) highlighted the need for easy-to-setup systems (installation time shorter than $15 \mathrm{~min}$ in $82 \%$ of responses) and revealed that the expected daily time of use would be higher than 2 hours. This, in particular, stresses the importance of mechanical comfort (selected as first priority for $72 \%$ of users and $60 \%$ of users) and customization of the application (second priority for $79 \%$ of users and $62 \%$ of medical staff). In terms of mechanical comfort, the main point was the compatibility with potential headrests and most cited applications were, in order of importance: voice synthesizer, web surfing, emails and domotics interfaces.

At the time of writing 4 ICU patients have been included in the feasibility study ( 2 male, 2 female, age range: 22 to 63 y.o.). Two patients presented with quadriplegia (C1, C4-C5), one with Guillain-Barre syndrome, and one was a post-surgery patient. Three patients were mechanically ventilated through translaryngeal tracheotomy (75\%). Even though the BCI spelling system was calibrated at a spelling rate of 1.8 characters per minute, post-analysis showed that two patients (50\%) had sufficient Signal-toNoise-Ratio (SNR) to achieved spelling rate comparable to those found in literature on healthy subjects. The other two patients withdrew from the study, one because of respiratory distress and the other because of an impossibility to set up occipital electrodes, respectively.

\subsection{Robust P300 paradigm}

Identification of optimal subset of sensors was carried out by comparing P300 identification performance from three different group of sensors: the complete EEG headset (32 electrodes), 8 electrodes individually selected to provide best performance, and common subgroup of 8 electrodes providing the best average performance in the population of 20 healthy volunteers $[19,20,21]$. This study, illustrated in Figure 2, shows that an individually customized subsets of 8 leads provides similar accuracy (Acc) than the complete headset (32 electrodes) in $85 \%$ of subjects. The subset of 8 electrodes (out of 32) common to the 20 subjects also provides equivalent performances, except for

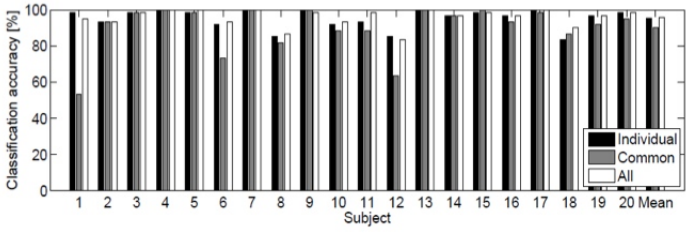

Figure 2. Description of classification accuracy for $n=20$ subjects + average (right) for all 32 sensors, an optimized subset of 8 sensors for all subjects and an individually optimized subset of sensors.

three patients (15\%) for whom the common subset of sensors does not provide sufficient accuracy for practical BCI use (Acc; $75 \%)$.

Optimization of the time window for P300 detection [22] and spatio-temporal feature selection [23] led to an average accuracy greater than $95 \%$ amongst the 20 subjects. In particular, partial use of training data provided an average accuracy of $80 \%$ with as little as 2 min signal for calibration [24]. This particularly meets the needs expressed by clinicians and patients for a short setup time.

Finally, the data set was used to understand better the implications of close target stimuli. Analysis of such events revealed that in order to elicit an unaltered P300, target stimuli should be separated by at least $450 \mathrm{~ms}$. A novel pseudo-random paradigm preventing such events, led to an estimated 30\% relative increase in bit-rate [25].

\subsection{Wireless EEG headset for daily use at home}

A prototype of the electronic board was designed. Thanks to the experimental setup based on the ECRINS board shown on Figure 3, the input referred noise of the recording system (based on a dedicated ASIC) and preliminary throughput tests have been performed. These first tests confirmed the feasibility of transmitting 32 channels with a 12-bit resolution sampled at $1 \mathrm{kHz}$. An input-referred RMS noise in BW1 $[0,5 \mathrm{~Hz} ; 300 \mathrm{~Hz}]$ of about $1 \mu \mathrm{V}$ for each channel was measured. A software for wireless recording and visualization of EEG signals was developed, and the complete system was validated in vivo on a rodent model, as described in Figure 1. The experimental procedures and animal care were carried out in compliance with the European Community Council Directive of 24th November 1986 (86/609/EEC).

Literature on dry electrode technology discarded micro-array technology for lack of sufficient evidence, and health and safety issues associated with minimally invasive modalities over time. Similarly, hybrid capacitive technology was discarded because of the necessary additional electronic stages, leaving comb designs the preferable technology for BCI. 


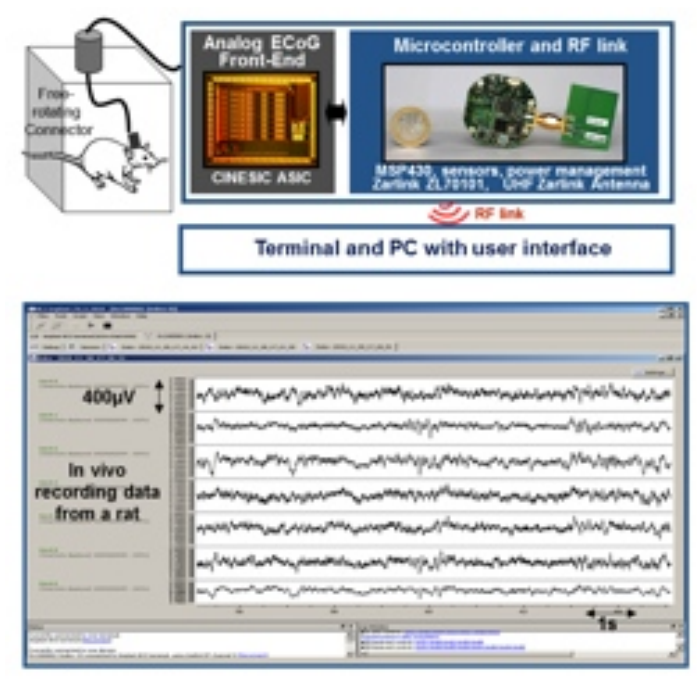

Figure 3. Description of the technical setting for the in vivo validation of the electronic card and the wireless link.

\section{Conlusion}

The current project will run for another year and a half, and should end with the clinical validation of the prototype in a population of severely disabled patients. The study will compare performances of the system with existing assistive technology. Major remaining steps involve the break down of line-columns stimuli in the P3Speller paradigm, development of an optimized version of the electronic board. The board will be connected to a headset specifically designed to provide comfortable and practical interface with dry electrodes.

The major advantage of the approach described here is the central role played by the clinical partner: from the original project design phase to the clinical validation, developments are led by pragmatic recommendations from practitioners and patients, allowing the know-how of engineers and researchers to benefit patients directly. Such a collaboration allowed us to address the issues encountered during the feasibility study at an early stage, and should remain the driving force behind this project.

In addition to the hardware and algorithms (available on the OpenViBE forge) developed in the scope of the project, benefits for the BCI community involve a first evaluation of patients' needs as well as patients' data acquired in Intensive Care Unit during a P300 speller protocol. Such an evaluation of patients' need and in-situ data are extremely rare in the BCI community that works mainly with healthy volunteers and is therefore highly valuable material to guide future research in the field.

Finally, if the system is proven to perform better than current users assistive technology in the clinical validation stage, commercial availability for severely disabled patients could be achieved within the next 5 years.

\section{Acknowledgement}

We would like to thank the National Research Agency (ANR) and the General Directorate for Armament (DGA) for funding this project (Project ANR- 09-TECS-013-01RoBIK). We would like to thank the medical staff who have been (and still are) involved on this project, and in particular: Aurelien Van Laghenhove, Michelle Lejaille, Marjorie Figère, Marine Garguillo from the CICIT; JeanMarie, Gilles and Caroline together with Dr. Azabou and Prof. Lofaso and Hérault from the Functional Exploration Unit, Justine Bouteille and Samuel Pouplin from the New Technologies Platform (PFNT), as well as many others involved on the project.

\section{References}

[1] J. Vidal, "Toward direct brain-computer communication," Annual review of Biophysics and Bioengineering, vol. 2, no. 1, pp. 157-180, 1973.

[2] N. Birbaumer, "Breaking the silence: brain-computer interfaces (bci) for communication and motor control," Psychophysiology, vol. 43, no. 6, pp. 517-532, 2006.

[3] N. Birbaumer and L. Cohen, "Brain-computer interfaces: communication and restoration of movement in paralysis," The Journal of physiology, vol. 579, no. 3, pp. 621-636, 2007.

[4] G. Schalk, D. McFarland, T. Hinterberger, N. Birbaumer, and J. Wolpaw, "Bci2000: a general-purpose brain-computer interface (bci) system," Biomedical Engineering, IEEE Transactions on, vol. 51, no. 6, pp. 1034-1043, 2004.

[5] Y. Renard, F. Lotte, G. Gibert, M. Congedo, E. Maby, V. Delannoy, O. Bertrand, and A. Lécuyer, "Openvibe: An open-source software platform to design, test, and use brain-computer interfaces in real and virtual environments," Presence: teleoperators and virtual environments, vol. 19, no. 1, pp. 35-53, 2010.

[6] L. Farwell and E. Donchin, "Talking off the top of your head: toward a mental prosthesis utilizing event-related brain potentials," Electroencephalography and clinical Neurophysiology, vol. 70, no. 6, pp. 510-523, 1988.

[7] S. Halder, M. Rea, R. Andreoni, F. Nijboer, E. Hammer, S. Kleih, N. Birbaumer, and A. K "ubler, "An auditory oddball brain-computer interface for binary choices," Clinical Neurophysiology, vol. 121, no. 4, pp. 516-523, 2010.

[8] L. Citi, R. Poli, C. Cinel, and F. Sepulveda, "P300based bci mouse with genetically-optimized analogue 
control," Neural Systems and Rehabilitation Engineering, IEEE Transactions on, vol. 16, no. 1, pp. 51-61, 2008.

[9] G. Muller-Putz and G. Pfurtscheller, "Control of an electrical prosthesis with an ssvep-based bci," Biomedical Engineering, IEEE Transactions on, vol. 55, no. 1, pp. 361-364, 2008.

[10] O. Bai, P. Lin, S. Vorbach, M. Floeter, N. Hattori, and M. Hallett, "A high performance sensorimotor beta rhythm-based brain-computer interface associated with human natural motor behavior," Journal of neural engineering, vol. 5, p. 24, 2008.

[11] D. Moran, "Evolution of brain-computer interface: action potentials, local field potentials and electrocorticograms," Current Opinion in Neurobiology, 2010.

[12] D. Krusienski, E. Sellers, D. McFarland, T. Vaughan, and J. Wolpaw, "Toward enhanced p300 speller performance," Journal of neuroscience methods, vol. 167, no. 1, pp. 15-21, 2008.

[13] B. Rivet, A. Souloumiac, V. Attina, and G. Gibert, "xdawn algorithm to enhance evoked potentials: application to brain-computer interface," Biomedical Engineering, IEEE Transactions on, vol. 56, no. 8, pp. 2035-2043, 2009.

[14] A. Rakotomamonjy and V. Guigue, "Bci competition iii: Dataset ii-ensemble of svms for bci p300 speller,' Biomedical Engineering, IEEE Transactions on, vol. 55, no. 3, pp. 1147-1154, 2008.

[15] L. Hutton, "Diamond microarray electrodes," 2009.

[16] A. Pradeep, R. Knight, and R. Gurumoorthy, "Dry electrodes for electroencephalography," Patent, Aug. 24, 2009, uS Patent App. 12/546,586.

[17] T. Zander, M. Lehne, K. Ihme, S. Jatzev, J. Correia, C. Kothe, B. Picht, and F. Nijboer, "Frontiers: A dry eeg-system for scientific research and braincomputer interfaces," Frontiers in Neuroprosthetics, vol. 5, 2011.

[18] J. Estepp, J. Christensen, J. Monnin, I. Davis, and G. Wilson, "Validation of a dry electrode system for eeg," vol. 53, no. 18, pp. 1171-1175, 2009.

[19] H. Cecotti, B. Rivet, M. Congedo, C. Jutten, O. Bertrand, E. Maby, and J. Mattout, "Suboptimal sensor subset evaluation in a p300 brain-computer interface," 2010.

[20] B. Rivet, H. Cecotti, R. Phlypo, O. Bertrand, E. Maby, and J. Mattout, "Eeg sensor selection by sparse spatial filtering in p300 speller brain-computer interface," in Engineering in Medicine and Biology Society (EMBC), 2010 Annual International Conference of the IEEE. IEEE, 2010, pp. 5379-5382.
[21] H. Cecotti, B. Rivet, M. Congedo, C. Jutten, O. Bertrand, E. Maby, and J. Mattout, "A robust sensor-selection method for p300 brain-computer interfaces," Journal of Neural Engineering, vol. 8, p. 016001, 2011.

[22] H. Cecotti, R. Phlypo, B. Rivet, M. Congedo, E. Maby, and J. Mattout, "Impact of the time segment analysis for p300 detection with spatial filtering," 2010.

[23] N. Jrad and M. Congedo, "Spatio-temporal feature extraction and classification of event-related potentials," 7e Plateforme AFIA Association Grancaise pour l'Intelligence Artificielle Chambery, 16 au 20 mai 2011, p. 233.

[24] H. Cecotti, G. Saint Ellier, B. Rivet, M. Perrin, E. Maby, and J. Mattout, "Reducing calibration time for the p300 brain-computer interface speller," 2010.

[25] H. Cecotti and B. Rivet, "A pilot study for improving the graphical user interface of p300 based bcis," 2010. 